\title{
COULD THE DELETERIOUS EFFECT OF STRETCHING ONLY INFLUENCE SOCCER PLAYERS WITH BETTER PERFORMANCE IN THE VERTICAL JUMP?
}

original paper

( ) University School of Physical Education in Wroclaw

DOI: https://doi.org/10.5114/hm.2018.79736

\section{FABRICIO VASCONCELLOS ${ }^{1,2,3}$, RENATO MASSAFERRI ${ }^{2,4,5}$, MARCOS REIS ${ }^{6}$, DANIEL CARNEVALE ${ }^{1,3}$, PAULO GIL SALLES ${ }^{3}$, JOAO BRITO ${ }^{7}$}

${ }^{1}$ Graduation Program in Exercise and Sport Sciences, Rio de Janeiro State University, Rio de Janeiro, Brazil

${ }^{2}$ Laboratory of Physical Activity and Health Promotion, Rio de Janeiro State University, Rio de Janeiro, Brazil

${ }^{3}$ Laboratory of Football Studies, Rio de Janeiro State University, Rio de Janeiro, Brazil

${ }^{4}$ Graduation Program in Clinical and Experimental Pathophysiology, Rio de Janeiro State University,

Rio de Janeiro, Brazil

${ }^{5}$ Graduation Program in Human Factors Operational Performance, University of the Air Force, Rio de Janeiro, Brazil

${ }^{6}$ Department of Physical Education, Estácio College of Sergipe, Aracaju, Brazil

${ }^{7}$ Portugal Football School, Portuguese Football Federation, Lisbon, Portugal

\section{ABSTRACT}

Purpose. The aim of the study was to investigate the acute effects of high vs. low volume stretching exercises on vertical jump in youth male soccer players and verify if different performances at baseline could exert any influence on these effects.

Methods. Overall, 45 players (mean age, $14.0 \pm 0.7$ years) from the $1^{\text {st }}$ division of Rio de Janeiro state championship were invited to participate in the study. They were assessed for vertical jump and stretching over 3 days. On day 1 , they were tested for vertical jump after a conventional warm-up. On day 2, the group was randomly divided into 2 subgroups: one subgroup tested for vertical jump after low-volume stretching and the other subgroup tested for vertical jump after high-volume stretching. On day 3, a counterbalanced order of the stretching protocol was employed. Then, the baseline vertical jump scores were stratified in terciles to compare the effect of high-volume stretching between the groups.

Results. After high-volume stretching, a significant decrease in vertical jump was detected $(-25.8 \% ; p=0.01 ;$ partial $\left.\eta^{2}=0.63\right)$, while no differences were observed for low-volume stretching. Moreover, players with better performance in vertical jump ( $1^{\text {st }}$ tercile) demonstrated a greater reduction in jumping height after high-volume stretching than those of the $3^{\text {rd }}$ tercile $\left(1^{\text {st }}\right.$ vs. $3^{\text {rd }}$ tercile: $-41.1 \pm 3.6 \%$ vs. $\left.-21.7 \pm 6.9 ; p<0.05\right)$.

Conclusions. The results suggest that players with best vertical jump performance can be more sensitive to the alterations in muscle inhibition after high-volume stretching exercises.

Key words: soccer, static stretching, lower extremity, muscle power, motor performance

\section{Introduction}

In team sports, stretching is widely used as a warmup routine before any training session or match. Static stretching can be understood as moving of a limb to the end of its range of motion and keeping that position for more than 15 seconds [1]. Dynamic stretching con- sists of self-controlled and dynamics exercises within the range of motion for each specific joint [1].

The effects of stretching can be influenced by the type of exercise and the volume of application. The volume of application of stretching exercises refers to the duration of exercise in different quantitative scales (e.g. time of the exercise). High-volume stretching can

Correspondence address: Fabrício V.A. Vasconcellos, Institute of Physical Education and Sports, Laboratory of Football Studies, Rio de Janeiro State University, Rua São Francisco Xavier 524 / sala 8121F - Maracanã, Rio de Janeiro, RJ, Brazil, CEP: 20550-013, e-mail: fabricio.vasconcellos@uerj.br

Received: July 18, 2018

Accepted for publication: November 6, 2018

Citation: Vasconcellos F, Massaferri R, Reis M, Carnevale D, Salles PG, Brito J. Could the deleterious effect of stretching only influence soccer players with better performance in the vertical jump? Hum Mov. 2018;19(5)special/issue:23-28; doi: https://doi.org/10.5114/hm.2018.79736. 
F. Vasconcellos, R. Massaferri, M. Reis, D. Carnevale, P.G. Salles, J. Brito, Static stretching and soccer players

be considered as lasting many seconds longer than ordinarily (e.g., in soccer it is not usual for a player to make stretching exercises for more than 10 seconds). Low-volume stretching is exercise with the duration shorter than ordinarily.

In this context, studies have demonstrated that static stretching methods might have a deleterious effect on muscle power of the lower limbs [1-3]. For example, many studies have shown that subjects who implemented static stretching before exercise decreased running economy [1], and shorter static stretch duration time was associated with a reduction of the negative acute effect on muscle power [3].

In this sense, dynamic and combined stretching exercises in relation to static stretching promoted more acceleration and speed to soccer players [4]. Soccer players produce more force for a faster action [4]. Therefore, static stretching exercises are not suitable for their increment to power and speed performance. However, dynamic stretching cannot be suitable for players with shortened muscles in relation to acceleration and speed performance and risk of injury, too [4].

This effect may vary depending on the volume of stretching [5], by decreasing the reflex sensitivity of muscle spindles [6], $\alpha$-motor-neuron excitability [7, 8], or muscle stiffness [9]. Concomitantly, the effects of stretching on muscle power in the lower limbs have been categorized according to type, volume, and competitive level, e.g. university sports science students, nonprofessional and professional soccer players $[1,3]$.

Stretching exercises induce a deleterious effect on lower limb power in young subjects [2, 10], but submaximal speed running might not be adversely affected by prior static stretching [1]. Still, few studies investigated the impact of different volume of stretching on power performance of lower limbs [11-13]. Maximal strength decreased after static stretching, although static stretching improved flexibility compared with ballistic stretching. Thus, static stretching could not be recommended before performance in high sports practice (e.g. soccer) that requires high levels of strength in lower limbs [11], but many high-level soccer players prefer static stretching before soccer matches [14]. To the best of our knowledge, no study so far has investigated whether athletes with better performance on muscle power at baseline are more susceptible to the deleterious effects of stretching.

In this way, as soccer players believe that static stretching can constitute an injury risk prevention strategy [14], investigating static stretching effects about vertical jump performance could improve the offer of stimuli before training sessions and soccer matches.
Besides, comparing acute effects of high vs. low volume static stretching could help to decrease the negative impact of static stretching in the context of players' desire to perform this kind of stretching.

In order to face these questions, the aim of the present study was to investigate the acute effects of high vs. low volume stretching exercises on vertical jump in youth male soccer players and to verify if different performances at baseline could exert any influence on these effects.

\section{Methods}

\section{Participants}

The study involved 45 youth male soccer players (mean age, $14.0 \pm 0.7$ years): 3 goalkeepers, 13 backwards, 20 midfielders, and 9 forwards. They had more than 3 years of practice experience in young Brazilian soccer. The participants were randomly selected from the 9 teams that participated in the U-15 first division of Rio de Janeiro state championship in 2009.

\section{Design and procedures}

The testing was conducted on 3 different days separated by 48 hours. On day 1, anthropometry (stature, body mass, and skinfolds), medical anamnesis, and baseline vertical jump testing were completed. To minimize the learning effect of protocols, the sample was divided into 2 groups; on days 2 and 3, each group performed the stretching exercises with different volumes in a counterbalanced way. On day 2 , group A performed high-volume stretching exercises and group B lowvolume stretching exercise, and then vertical jump performance was evaluated. On day 3, the order was reversed. After that, the baseline vertical jump scores were stratified in terciles (the $1^{\text {st }}$ tercile represents the individuals with the best results - highest vertical jump, and the $3^{\text {rd }}$ tercile represents subjects with the worst results - lowest vertical jump) to compare the effect of high-volume stretching between the groups.

All procedures were performed by the team's physical coach. The data were collected during the competitive season in the second half of the year during the Brazilian winter (temperature $18-28^{\circ} \mathrm{C}$ ). The participants were playing the regional championship with one official game per week. 


\section{Measures}

\section{Vertical jump}

The evaluation of the vertical jump was performed on a jumping platform (Jumptest ${ }^{\circledR}$, Hidrofit LTDA, Brasil). The equipment had been validated elsewhere $[15,16]$. Then, the participants were oriented to stand with the feet on the jumping platform and jump as high as possible with the hands akimbo and total freedom of movement of hip, knee, and ankle joints [17]. Three trials of the vertical jump were conducted, with 45-second rest interval between the sets. The best result was adopted as the vertical jump maximal performance.

\section{Stretching}

The static stretching exercises for gluteus, hamstrings, quadriceps, and gastrocnemius are presented in Table 1. All participants were instructed to slowly reach the point of greatest joint amplitude and keep on this position. For low-volume stretching, this position was maintained during 1 set of 10 seconds in each position. For high-volume stretching, the position was maintained during 3 sets of 20 seconds in each position. The soccer players only performed a warm-up protocol consisting of 10-minute treadmill running at $8 \mathrm{~km} / \mathrm{h}$ before the stretching protocol, that is, they performed the warm-up right after the static stretching exercises and for last they underwent the vertical jump assessment.

There were performed 1 set (repetition) of 10 seconds and 3 sets of 20 seconds of stretching protocol because, generally in sports, especially in soccer, players do not use more than 10 seconds per static stretching

Table 1. Description of stretching exercises conducted prior to vertical jump testing

(A) Dorsiflexion of the ankle with hip and knee extended: the soccer players were with the hand on the tip of the toe with the hip and knee extended and stabilized

Dorsal decubitus

(B) Hip flexion with extended knee: the players performed leg raising (one-sided) without flexing their knees

(C) Hip and knee flexion: the subjects raised the legs with flexing hip and knees

(D) Hip extension and knee flexion:

Ventral decubitus the participants sustained their hands on the ground while they did hip extension and knee flexion exercise. This way, these 2 protocols were implemented because they are actually very popular in soccer training (anecdotal report). In this sense, the stretching protocol of 3 sets of 20 seconds was treated as 'high-volume stretching' because it lasted 6 times longer than the stretching protocol of 1 set of 10 seconds (which it is not usual in soccer because the time is smaller).

\section{Statistical analyses}

For the data analyses, descriptive and inferential techniques were used. The Kolmogorov-Smirnov test verified the normality of the data. To test the differences between the stretching volume groups, one-way ANOVA for repeated measures was applied, while twoway ANOVA for repeated measures and Tukey-Kramer post-hoc test served to detect the differences between the variation baseline vs. post-stretching with different volumes on terciles. The statistical significance of the results was set at $p<0.05$. Subsequently, the effect size relative to the comparison among 3 or more variables was established through the partial $\eta^{2}$ for the effect of volume, and the effect size among terciles was verified with Cohen's $d$ test [18].

\section{Ethical approval}

The research related to human use has been complied with all the relevant national regulations and institutional policies, has followed the tenets of the Declaration of Helsinki, and has been approved by the authors' institutional review board or an equivalent committee.

\section{Informed consent}

Informed consent has been obtained from the legal guardians of all individuals included in this study. The participating clubs signed an information term of responsibility, in accordance with the ethical criteria for research as approved by the local ethics committee (0178/2008).

\section{Results}

Table 2 shows anthropometric characteristics and the vertical jump performance in the terciles. Significant differences were detected between the $1^{\text {st }}$ (the highest) and the $3^{\text {rd }}$ (the lowest) terciles on vertical jump.

Figure $1 \mathrm{~A}$ shows a significant decrease in the vertical jump performance after high-volume stretching $\left(\mathrm{F}_{[2,46]}=9.44 ; p=0.01 ;\right.$ partial $\left.\eta^{2}=0.63\right)$. No significant differences were detected after low-volume stretching. When the terciles were compared, a great loss of per- 
F. Vasconcellos, R. Massaferri, M. Reis, D. Carnevale, P.G. Salles, J. Brito, Static stretching and soccer players

Table 2. Anthropometric characteristics and vertical jump performance in terciles at baseline

\begin{tabular}{lrrrr} 
& Mean $\pm S D$ & $1^{\text {st }}$ tercile & $2^{\text {nd }}$ tercile & $3^{\text {rd }}$ tercile \\
\hline Age (years) & $14.9 \pm 0.7$ & $14.7 \pm 0.2$ & $14.3 \pm 0.8$ & $14.6 \pm 0.5$ \\
Body mass (kg) & $59.5 \pm 6.5$ & $57.6 \pm 7.2$ & $60.3 \pm 5.1$ & $59.1 \pm 9.6$ \\
Stature (cm) & $168.4 \pm 7.4$ & $169.6 \pm 10.2$ & $167.2 \pm 4.9$ & $168.3 \pm 6.7$ \\
BMI (kg/cm $\left.{ }^{2}\right)$ & $21.0 \pm 1.4$ & $20.0 \pm 2.0$ & $21.6 \pm 1.0$ & $20.9 \pm 1.4$ \\
Vertical jump (cm) & $33.36 \pm 3.6$ & $39.54 \pm 3.9$ & $34.06 \pm 2.6$ & $30.03 \pm 2.0 *$ \\
\hline
\end{tabular}

* significant difference for the $1^{\text {st }}$ tercile, $p=0.03$

A

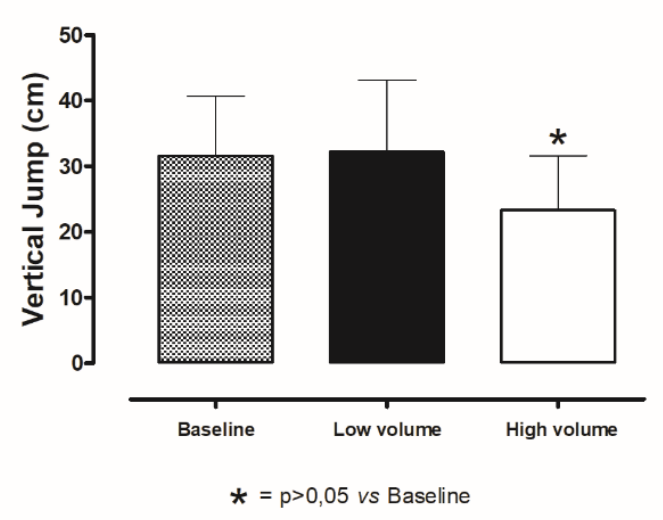

B

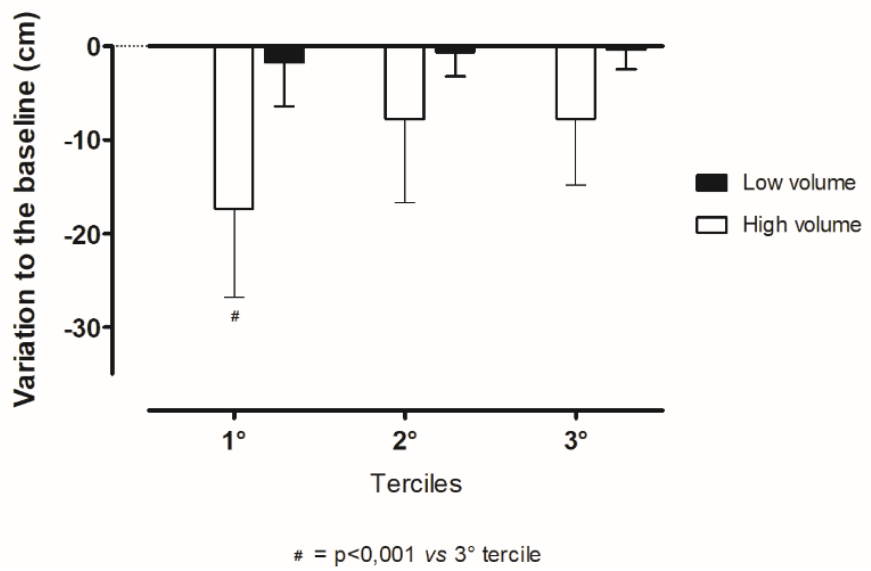

Figure 1. (A) Overall effects of volume of stretching on vertical jump performance. (B) Comparisons among terciles of vertical jump performance depending on the volume of stretching

formance after high-volume stretching was observed in the $1^{\text {st }}$ tercile compared with the $3^{\text {rd }}$ tercile $\left(1^{\text {st }}\right.$ vs. $3^{\text {rd }}$ tercile: $-41.1 \pm 3.6 \%$ vs. $-21.7 \pm 6.9$, respectively; $p<0.05 ; d=2.25$; Figure 1B).

\section{Discussion}

The main finding of the present study is that subjects with better vertical jump performance decreased their execution in vertical jump after high volume stretching exercise. These results may be related with the time of high volume stretching exercises. High volume training might have stimulated the Golgi tendon organs, decreasing agonist muscle action, and, consequently, causing the vertical jump difference [19].

Other studies reported decreases in strength after passive and static stretching in the quadriceps [20]. Behm et al. [20] tested 5 sets of 45 seconds of stretching exercise in 12 active men. The tests were performed before and 6 and 10 minutes after the stretching exercises. Each subject conducted 3 maximal isometric contractions of the quadriceps. The maximum muscle contraction decreased by $12 \%$ after stretching. Stretching may have reduced the maximal contraction owing to muscle inhibition rather than turning muscle units more complacent [20,21].
Mariscal et al. [22] did not find any negative effect in the countermovement jump, Abalakov jump, or the 40m linear sprint performance after static stretching exercises. However, static stretching exercises were performed in time smaller than 10 seconds. High-level soccer players believe static stretching before soccer matches could prevent injury [14]; this information is important because a low-volume static stretching does not result in risk of injury or performance decrease [22], as observed in our study (in relation to the vertical jump performance).

To our knowledge, this is the first study that evaluated whether the subjects with best results in vertical jump testing had a greater decrease in vertical jump performance. The differences among terciles with regard to performance may be attributed to the fact that the subjects with best performance can be more sensitive to the alterations in muscle inhibition after high volume stretching exercise [23]. Given the plethora of stretching methods and contradictory results [1, 24-26], more studies are needed to test the preliminary data presented here.

In addition to the impairment of strength performance, static stretching can enhance the injury risk in players. Baumgart et al. [27] found that static stretching exercises before a soccer match increased anterior tibi- 
al translation of the knee joint. In this way, the physical coach should understand the negative effects of this practice (concerning both the performance and injury prevention) in order to perform functional and specific exercises in the warm-up before the matches and training, and also to make players aware about the negative impact of this practice in the preparation for games.

The practical applications of these results will assist coaches in planning before games and training sessions by choosing specific exercises and respecting the players' desire to perform static stretching that is generally low-volume when performed spontaneously. A limitation of this study was the difficulty to find reference values for load volume prescription of static stretching exercises because soccer players generally do not practise them for more than 10 seconds naturally.

\section{Conclusions}

The present investigation concludes that high-volume stretching exercise negatively affects vertical jump performance in youth male soccer players. Besides, players with best vertical jump performance are more negatively affected by high-volume stretching exercise. Regarding the practical application, these findings are extremely relevant because they can contribute to players' sports performance through improved daily training with the modulation of stretching exercises.

\section{Acknowledgements}

This study was partially supported by grants from the Carlos Chagas Filho Foundation for Research Support in Rio de Janeiro (FAPERJ) and the Brazilian National Council for Scientific and Technological Development (CNPq).

\section{Disclosure statement}

No author has any financial interest or received any financial benefit from this research.

\section{Conflict of interest}

The authors state no conflict of interest.

\section{References}

1. Behm DG, Chaouachi A. A review of the acute effects of static and dynamic stretching on performance. Eur J Appl Physiol. 2011;111(11):2633-2651; doi: 10.1007/ s00421-011-1879-2.

2. Chaouachi A, Castagna C, Chtara M, Brughelli M, Turki O, Galy O, et al. Effect of warm-ups involving static or dynamic stretching on agility, sprinting, and jump- ing performance in trained individuals. J Strength Cond Res. 2010;24(8):2001-2011; doi: 10.1519/JSC.0b 013e3181aeb181.

3. Simic L, Sarabon N, Markovic G. Does pre-exercise static stretching inhibit maximal muscular performance? A meta-analytical review. Scand J Med Sci Sports. 2013;23(2):131-148; doi: 10.1111/j.1600-0838.2012. 01444.x.

4. Amiri-Khorasani M, Calleja-Gonzalez J, MogharabiManzari M. Acute effect of different combined stretching methods on acceleration and speed in soccer players. J Hum Kinet. 2016;50(1):179-186; doi: 10.1515/ hukin-2015-0154.

5. Feland JB, Myrer JW, Schulthies SS, Fellingham GW, Measom GW. The effect of duration of stretching of the hamstring muscle group for increasing range of motion in people aged 65 years or older. Phys Ther. 2001;81(5): 1110-1117.

6. Avela J, Kyröläinen H, Komi PV. Altered reflex sensitivity after repeated and prolonged passive muscle stretching. J Appl Physiol. 1999;86(4):1283-1291; doi: 10.1152/jappl.1999.86.4.1283.

7. Garland SJ. Role of small diameter afferents in reflex inhibition during human muscle fatigue. J Physiol. 1991; 435(1):547-558; doi: 10.1113/jphysiol.1991.sp018524.

8. Gandevia SC, McKenzie DK. Activation of human muscles at short muscle lengths during maximal static efforts. J Physiol. 1988;407(1):599-613; doi: 10.1113/ jphysiol.1988.sp017434.

9. Marek SM, Cramer JT, Fincher AL, Massey LL, Dangelmaier SM, Purkayastha S, et al. Acute effects of static and proprioceptive neuromuscular facilitation stretching on muscle strength and power output. J Athl Train. 2005;40(2):94-103.

10. Paradisis GP, Pappas PT, Theodorou AS, Zacharogiannis EG, Skordilis EK, Smirniotou AS. Effects of static and dynamic stretching on sprint and jump performance in boys and girls. J Strength Cond Res. 2014;28(1):154160; doi: 10.1519/JSC.0b013e318295d2fb.

11. Bacurau RF, Monteiro GA, Ugrinowitsch C, Tricoli V, Cabral LF, Aoki MS. Acute effect of a ballistic and a static stretching exercise bout on flexibility and maximal strength. J Strength Cond Res. 2009;23(1):304308; doi: 10.1519/JSC.0b013e3181874d55.

12. Fletcher IM, Anness R. The acute effects of combined static and dynamic stretch protocols on fifty-meter sprint performance in track-and-field athletes. J Strength Cond Res. 2007;21(3):784-787; doi: 10.1519/R-19475.1.

13. Dalrymple KJ, Davis SE, Dwyer GB, Moir GL. Effect of static and dynamic stretching on vertical jump performance in collegiate women volleyball players. J Strength Cond Res. 2010;24(1):149-155; doi: 10.1519/ JSC.0b013e3181b29614.

14. Zech A, Wellmann K. Perceptions of football players regarding injury risk factors and prevention strategies. PLoS One. 2017;12(5):e0176829; doi: 10.1371/ journal.pone.0176829. 


\section{HUMAN MOVEMENT}

F. Vasconcellos, R. Massaferri, M. Reis, D. Carnevale, P.G. Salles, J. Brito, Static stretching and soccer players

15. Ferreira JC, Carvalho RGS, Szmuchrowski LA. Validity and reliability of a contact tape for vertical jump height measurement [in Portuguese]. Braz J Biomech. 2008; 9(17):93-99.

16. De Salles PG, Vasconcellos FV, de Salles GF, Fonseca RT, Dantas EH. Validity and reproducibility of the sargent jump test in the assessment of explosive strength in soccer players. J Hum Kinet. 2012;33(1):115-121; doi: 10.2478/v10078-012-0050-4.

17. McCann MR, Flanagan SP. The effects of exercise selection and rest interval on postactivation potentiation of vertical jump performance. J Strength Cond Res. 2010;24(5):1285-1291; doi: 10.1519/JSC.0b013e3181d 6867c.

18. Espírito-Santo H, Daniel F. Calculating and reporting effect sizes on scientific papers (1): $p<0.05$ limitations in the analysis of mean differences of two groups [in Portuguese]. Port J Behav Social Res. 2015;1(1):3-16; doi: 10.7342/ismt.rpics.2015.1.1.14.

19. Jaggers JR, Swank AM, Frost KL, Lee CD. The acute effects of dynamic and ballistic stretching on vertical jump height, force, and power. J Strength Cond Res. 2008;22(6):1844-1849; doi: 10.1519/JSC.0b013e3181 $854 \mathrm{a} 3 \mathrm{~d}$

20. Behm DG, Bambury A, Cahill F, Power K. Effect of acute static stretching on force, balance, reaction time, and movement time. Med Sci Sports Exerc. 2004;36(8): 1397-1402; doi: 10.1249/01.MSS.0000135788.23012.5F.

21. Avloniti A, Chatzinikolaou A, Fatouros IG, Avloniti C, Protopapa M, Draganidis D, et al. The acute effects of static stretching on speed and agility performance depend on stretch duration and conditioning level. J Strength Cond Res. 2016;30(10):2767-2773; doi: 10.1519/JSC. 0000000000000568.

22. Mariscal SL, Garcia VS, Fernández-García JC, Saéz de Villarreal E. Acute effects of ballistic vs passive static stretching involved in a pre-match warm-up regarding vertical jump and linear sprint performance in soccer players. J Strength Cond Res. 2018; doi: 10.1519/JSC. 0000000000002477.

23. Kallerud H, Gleeson N. Effects of stretching on performances involving stretch-shortening cycles. Sports Med. 2013;43(8):733-750; doi: 10.1007/s40279-0130053-x.

24. Saez Saez de Villarreal E, González-Badillo JJ, Izquierdo M. Optimal warm-up stimuli of muscle activation to enhance short and long-term acute jumping performance. Eur J Appl Physiol. 2007;100(4):393-401; doi: 10.1007/s00421-007-0440-9.

25. Samuel MN, Holcomb WR, Guadagnoli MA, Rubley MD, Wallmann H. Acute effects of static and ballistic stretching on measures of strength and power. J Strength Cond Res. 2008;22(5):1422-1428; doi: 10.1519/JSC.0b013e $318181 \mathrm{a} 314$.

26. Donti O, Tsolakis C, Bogdanis GC. Effects of baseline levels of flexibility and vertical jump ability on performance following different volumes of static stretching and potentiating exercises in elite gymnasts. J Sports Sci Med. 2014;13(1):105-113.

27. Baumgart C, Gokeler A, Donath L, Hoppe MW, Freiwald J. Effects of static stretching and playing soccer on knee laxity. Clin J Sport Med. 2015;25(6):541-545; doi: 10.1097/JSM.0000000000000174. 